\title{
Abstract \\ Association between the pre-operative over-fasting and intra operative vomiting in pediatric anesthetic practice
}

\author{
Jayasinghe AVK ${ }^{1}$, Sooriyaarachchi $\mathrm{CMD}^{2}$, Jayalath $\mathrm{JAJU}^{2}$, Karunarathne YAWA ${ }^{2}$ \\ ${ }^{1}$ Postgraduate Institute of Medicine, ${ }^{2}$ National Institute of Health Sciences
}

Key words: Disaster management, knowledge, practices, associated factors, teachers

\begin{abstract}
Introduction
Not adhering to proper standards of fasting guidelines and subsequent over-fasting are known to cause intra-operative vomiting which may lead to severe complication like pulmonary aspiration.
\end{abstract}

Objective

To determine the association between pre-operative over-fasting and intra-operative vomiting among children who were undergoing bone marrow aspiration under general anesthesia at a tertiary care setting in Sri Lanka.

Methods

A prospective cohort study was done from May to December 2015 at National Cancer Institute Maharagama on children who were undergoing bone marrow aspiration with general anesthesia. The average fasting time of the exposed group is 5 hours following last clear fluid intake. The average fasting time of the Non-exposed group is 2 hours and 30 minutes. Calculated sample size was 202 participants for each group. Both groups were followed up for 6 hours following induction of anesthesia. Study instruments included an interviewer-administered questionnaire and a data extraction sheet.

Results

The response rate was $100 \%$. The median (IQR) age of the exposed and nonexposed groups were $6(4-9)$ and $5(4-7)$ respectively. 32 out of 202 children $(15.8 \%)$ in exposed group developed intra-operative vomiting while 17 out of 202 children $(8.4 \%)$ in non exposed group developed intra-operative vomiting. Relative risk of developing intra-operative vomiting among over-fasting children was 1.88 $(95 \%$ C.I. $=1.08$ to 3.28$)$.

\section{Conclusions and Recommendations}

Over-fasting is a significant risk factor for developing intra-operative vomiting in pediatric anesthetic practice. Measures must be taken to promote the correct fasting adherence in pediatric surgery.

Corresponding Author: AVK Jayasinghe, Email:< vidura.jayasinghe@yahoo.com>

Presentation at the $5^{\text {th }}$ biennial academic sessions of the Postgraduate institute of Medicine on 06 October 2016 Competing Interests: Authors have declared that no competing interests exist. 